\title{
PERCEPTIONS DU CHANGEMENT CLIMATIQUE ET PRATIQUES ADAPTATIVES DES AGRICULTEURS DU SAIS (MAROC)
}

\author{
Larbi Aziz* iD \\ Mohammed Mahdi** iD \\ Asmae Baba Khouya*** iD
}

\section{RESUMÉ}

Cet article traite des perceptions des changements climatiques, de leurs effets sur l'activité agricole et des pratiques adaptatives des agriculteurs de la commune rurale de bittit (province d'el hajeb au maroc). L'analyse des résultats, des «entretiens de groupes» et des «enquêtes par questionnaire» réalisés auprès d'un échantillon d'agriculteurs montre que les agriculteurs sont conscients du changement climatique. Elle révèle quoi l'univers de perception des changements de ces agriculteurs, est structuré autour de trois facteurs: la pluviométrie, la température et l'enneigement. Ces facteurs, ffectent les systèmes de production agricole et les agriculteurs réagissent en développant des pratiques adaptatives.

Mots-clés: agriculture, changement climatique, Maroc, perceptions, pratiques adaptatives, sais.

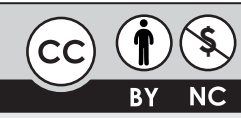

Recibido: 3 de noviembre de 2019 | Aprobado: 7 de febrero de 2020.

CÓMO CITAR ESTE ARTÍCULO:

Aziz, L., Mahdi, M., y Baba, A. (2020). Perceptions du changement climatique et pratiques adaptatives des agriculteurs du Sais (Maroc). Collectivus, Revista de Ciencias Sociales, 7 (2), 19-33. DOI: https://doi.org/10.15648/Collectivus.vol7num2.2020.2671

\footnotetext{
"Docteur en sociologie de l'environnement. École Nationale d'Agriculture de Meknès, Département d'Ingénierie du Développement, B.P. S/ 40, Meknès (Maroc).laziz@enameknes.ac.ma

"Docteur en Sociologie rurale. École Nationale d'Agriculture de Meknès, B.P. S/ 40, Meknès (Maroc).mahdi@enameknes.ac.ma

${ }_{* * *}^{*}$ Ingénieure agronome. École Nationale d'Agriculture de Meknès, Département d'Ingénierie du Développement, B.P. S/ 40, Meknès (Maroc). asma.baba18@gmail.com
} 
20| Perceptions du changement climatique et pratiques adaptatives des agriculteurs du Sais (Maroc)

\title{
PERCEPCIONES DEL CAMBIO CLIMÁTICO Y PRÁCTICAS ADAPTATIVAS DE LOS AGRICULTORES SAIS (MARRUECOS)
}

\section{RESUMEN}

Este artículo analiza las percepciones del cambio climático, sus efectos en la actividad agrícola y las prácticas de adaptación de los agricultores en la comuna rural de Bittit (provincia de El Hajeb, Marruecos). El análisis de los resultados, de las "entrevistas grupales" y en las "encuestas de cuestionarios" realizadas entre una muestra de agricultores, da cuenta que los agricultores son conscientes del cambio climático. Revela que el universo de percepción, de los cambios de estos agricultores se estructura en torno a tres factores: las precipitaciones, la temperatura y la nieve. Estos factores, afectan los sistemas de producción agrícola y los agricultores responden desarrollando prácticas adaptativas.

Palabras clave: agricultura, cambio climático, Marruecos, percepciones, prácticas adaptativas, Sais.

\section{CLIMATE CHANGE PERCEPTIONS AND ADAPTIVE PRACTICES OF SAIS FARMERS (MOROCCO)}

\begin{abstract}
This article discusses perceptions of climate change, their effects on agricultural activity and the adaptive practices of farmers in the rural commune of Bittit (El Hajeb province, Morocco). Analysis of the results of "group interviews" and "questionnaire surveys" conducted among a sample of farmers shows that farmers are aware of climate change. It reveals that the universe of perception of the changes of these farmers is structured around three factors: rainfall, temperature and snow. These factors affect agricultural production systems and farmers respond by developing adaptive practices.
\end{abstract}

Keywords: adaptive practices, agriculture, climate change, Morocco, perceptions, Sais.

\section{Introduction}

Les changements climatiques, survenus dans le globe se manifestent par des sécheresses répétitives, des pluies diluviennes provoquant des inondations, et produisent des incidences économiques, environnementales et sociales négatives pour la vie de l'humanité. Ces changements et leurs impacts sont désormais reconnus comme l'un des plus grands défis du monde, de ses peuples, de son environnement et de ses économies. Mais ce sont les pays et les populations vulnérables qui en sont les plus affectées.

Le Groupe d'experts intergouvernemental sur l'évolution du climat (GIEC, 2014) a démontré que les activités humaines sont responsables des émissions massives de gaz à effet de serre (GES) dans l'atmosphère et l'accroissement des températures. Mais les pays les plus touchés par ces effets ne sont et ne seront pas nécessairement les plus responsables (Bertrand et Richard, 2012). C'est bien le cas des pays du continent africain qui, bien qu'ils n'émettent que 3,4\% des émissions mondiales de GES, ils sont, par contre et paradoxalement, les plus exposés aux effets des changements climatiques. Au premier chef de ces changements, l'augmentation des températures et la réduction de la pluviométrie qui impactent négativement leurs activités agricoles (GIEC, 2013). 
Ces changements augmentent l'aridité des sols, réduisent les rendements agricoles (FAO, 2013) et perturbent profondément les cycles des cultures (CIRAD, 2009). L'agriculture qui est la base de l'économie de ces pays s'en trouve bouleversée. Le changement climatique est désormais une réalité avérée globalement à l'échelle de la planète. ${ }^{1}$ Dans le même temps, et à des échelles locales, les disciplines des sciences sociales cherchent, entre autres, à comprendre et à analyser les perceptions et les comportements des populations locales face au changement climatique. Elles mettent au point des outils pour les détecter et les mesurer (Rishi et Mudaliar, 2014). Dans ce sens, Mike Hulme exhorte à adopter un regard très attentif sur le local, sur la dimension quotidienne et sur l'expérience individuelle du changement climatique (Breda, 2019). Nombreux chercheurs se sont intéressés aux percceptions de ce changement par les agriculteurs, et à s'informer sur leurs pratiques pour s'y adapter (Vissoh et al, 2012, Dugué, 2012, Aziz et Sadok, 2015, Aziz et Elquaoumi, 2016). L'adaptation au changement climatique est, désormais, moins un choix qu'une nécessité pour les agriculteurs des pays qui en sont les plus touchés (GIEC, 2014).

Cet article apporte une preuve supplémentaire, à partir d'un cas d'agriculteurs marocains, que: i) les agriculteurs sont conscients des changements climatiques et de leurs effets sur leurs activités agricoles, ii) leurs perceptions de ces changements proviennent en premier chef de leur expérience empirique et ils les expriment à travers des manifestations de changement concrètes et, iii) ils mettent en œuvre des pratiques nouvelles pour s'adapter à ces changements.

\section{Méthodologie}

La recherche à la base de cet article s'est intéressée aux aspects cognitifs et aux pratiques du changement climatique chez les agriculteurs d'une petite zone agricole, la commune rurale Bittit relevant de la province d'El Hajeb, région de Fès-Meknès? L'objectif est de contribuer, à partir d'une étude de cas, à la connaissance de l'univers local des perceptions des changements climatiques de l'agriculteur marocain. La recherche se place à l'échelle locale et au niveau de l'expérience personnelle des agriculteurs pour inventorier leurs perceptions des changements climatiques, les effets de ces changements sur leurs systèmes de production et les pratiques qu'ils développent pour s'y adapter ou atténuer leurs impacts.

Le climat de la zone d'étude est semi-aride. Concernant la pluviométrie, la moyenne annuelle des précipitations est de $412 \mathrm{~mm}$. La saison pluvieuse s'étale de l'automne jusqu'au début du printemps, avec des maximums en décembre et février. La température minimale est de $-2^{\circ} \mathrm{C}$ atteinte généralement entre Janvier et Février, alors que la maximale se situe aux alentours de $45^{\circ} \mathrm{C}$, atteinte en Août.

\footnotetext{
${ }^{1}$ Sauf pour les climato sceptiques. Dans un article publié dans la revue "Climatic Change", en 2013, le sociologue américain Robert Brulle démontre la collusion entre climato scepticisme et les groupes industriels et financiers qui fiancent des organisations de toutes sortes qui entretiennent le doute sur la réalité du réchauffement anthropique. http://www.lemonde.fr/ planete/ article/ 2013/12/31/les-climatosceptiques-qui-valaient-des-milliards_4341572_3244.html.

$2 \mathrm{Au}$ Maroc, le découpage administratif fait état de 12 régions dont celle de Fès-Meknès qui est, principalement, à vocation agricole (la plaine de Saiss).
} 
Figure 1. Situation de la zone d'étude

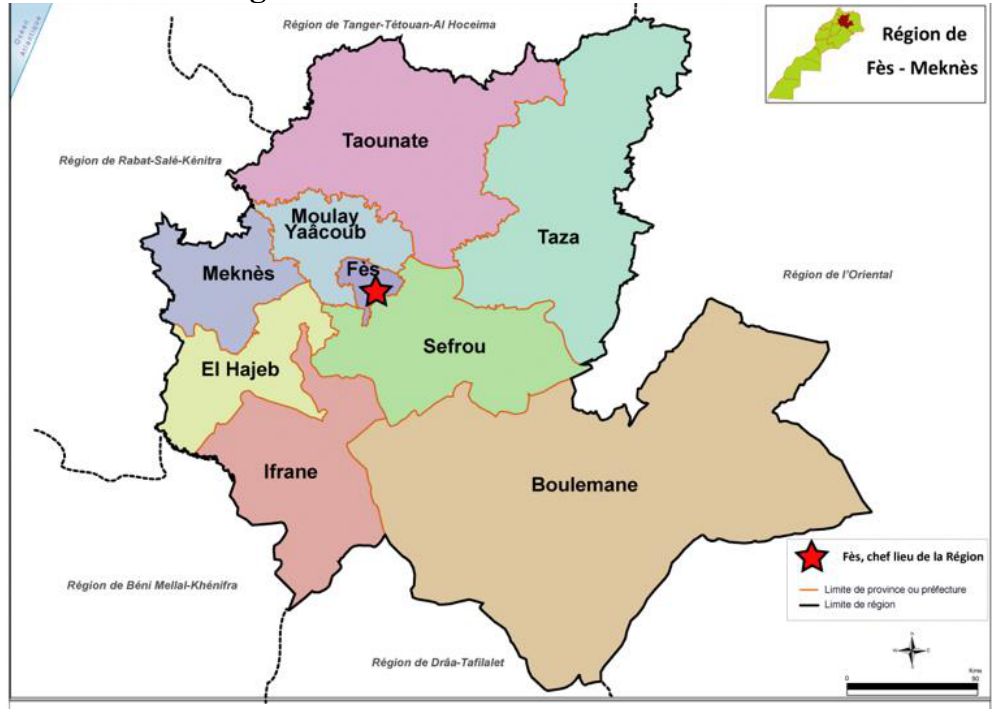

Source: Ministère de l'Intérieur (2015)

Pour collecter les données deux types d'entretien ont été conduits. Dans un premier temps, des entretiens semi-structurés réalisés avec des agriculteurs de divers Douars (villages) de la commune rurale Bittit, pour appréhender les dimensions de leur perception du changement climatique et leurs pratiques adaptatives. ${ }^{3}$ Dans un deuxième temps, des entretiens individuels par questionnaire standardisé, administré à une cinquantaine d'agriculteurs d'Ait Amer, l'un des sept Douars de la commune, pour recueillir les opinions des agriculteurs sur les dimensions de perception du changement révélées par les entretiens de groupe et leur lien avec les pratiques adaptatives.

Les données recueillies ont été soumises à une analyse descriptive pour les données quantitatives et à une analyse de contenu pour les données qualitatives.

\section{Résultats et discussion}

Les agriculteurs enquêtés vivent dans un même Douar, qui est l'unité de base de l'organisation sociale, économique et territoriale dans le monde rural marocain, et forment avec d'autres agriculteurs non enquêtés une collectivité ethnique et territoriale à base tribale caractérisée par des liens d'interconnaissance et d'échanges économiques, matrimoniaux et d'informations très marqués. Le Douar partage la même histoire et la même culture ${ }^{4}$. Les agriculteurs enquêtés sont des chefs d'exploitations agricoles familiales qui vivent de l'agriculture et de l'élevage. Ils représentent toutes les classes d'âge, qui varient entre 33 et 84 ans, avec une moyenne de 58 ans. Dans cette population,

${ }^{3}$ L'article valorise les données d'un Projet de Fin d'Études réalisé dans le cadre du projet PRIMS, qui porte sur l'intégration des petites et moyennes exploitations agricoles (PMEA) au marché dans le Saïs (Maroc). École Nationale d'Agriculture de Meknès.

4 Ces caractéristiques de la population enquêtée donnent à leurs perceptions des changements climatiques le caractère de «représentations sociales» que Jodelet D. (1989: 49) définit à la suite de Durkheim E. (1895) et Moscovici S. (1961) comme «une forme de connaissance, socialement élaborée et partagée, ayant une visée pratique et concourant à la construction d'une réalité 
$42 \%$ est âgé de 60 ans et plus, et dispose d'un recul historique pour apprécier les changements survenus dans son territoire. Les jeunes représentent $20 \%$ de l'échantillon; leurs appréciations du changement n'ont pas été négligés. Le niveau d'instruction de la population enquêtée est assez faible, puisque seulement 14\% justifient d'une scolarité secondaire ou universitaire. Le reste se répartit entre les niveaux d'instruction coranique ou primaire. Les analphabètes représentent $26 \%$. Les acquis de l'expérience vécue et le niveau d'étude influent largement sur leurs perceptions du changement climatiques, comme il sera montré par la suite.

Ce sont des agriculteurs qui exploitent des petites superficies réparties en trois catégories: les micro-exploitations (moins de 3 ha), les petites exploitations ( 3 à 5 ha) et les moyennes exploitations ( 5 à 20 ha). Presque la totalité des terres exploitées sont irriguées à partir de puits. Les perceptions du changement, dont il sera question, seraient celles d'agriculteurs vivant dans une région dite de «Bour ${ }^{5}$ favorable» et située sous un climat subhumide, qui a évolué vers un climat semi-aride. Les entretiens de groupe ont révélé que le mot sécheresse, par exemple, n'était pas utilisé par le passé dans la région contrairement à d'autres sociétés rurales marocaines qui ont toujours vécues avec la sécheresse et qui la désigne par un vocable spécial, Jdoub (Kamil, 1999). Le statut juridique des terres dominant est le privé «Melk», présent chez $82 \%$ des exploitants enquêtés. Une partie de ces terres Melk est en situation d'indivision. Le statut collectif régit les $18 \%$ des terres restant. Le foncier est assez sécurisé malgré l'existence du collectif et de l'indivision qui, en principe, fragilisent le développement de l'activité agricole.

\subsection{Perceptions des manifestations du changement climatique}

«Le climat est l'ami proche de l'agriculteur» affirme un enquêté. Le changement climatique dans la région est une réalité évidente et perceptible à l'œil nue pour $74 \%$ des agriculteurs enquêtés. Le climat et ses variations est une préoccupation quotidienne qu'il devient aisé aux agriculteurs d'en constater les changements importants. Cet avis se retrouve chez les agriculteurs plus au moins âgés. Les plus jeunes, par contre, ont pris connaissance du changement climatique à travers les mass médias. Le changement climatique est discernable à partir de plusieurs manifestations. Les plus remarquables sont la diminution de la pluviométrie et l'arrivée précoce de l'été (Tb. 1).

Tableau 1. Manifestations du changement climatique

\begin{tabular}{|l|c|c|}
\hline \multicolumn{1}{|c|}{ Manifestations du changement climatique } & Nombre de réponses & Pourcentage \\
\hline Diminution des quantités de pluie & 37 & $41 \%$ \\
\hline Arrivée précoce de l'été & 32 & $35 \%$ \\
\hline Perturbation des saisons & 10 & $11 \%$ \\
\hline Irrégularité de la pluviométrie & 5 & $6 \%$ \\
\hline Arrivée tardive des pluies & 4 & $4 \%$ \\
\hline Incertitude de voir la pluie arriver & 3 & $3 \%$ \\
\hline Total & 91 & $100 \%$ \\
\hline
\end{tabular}

Source: Enquêtes, 2017.

\footnotetext{
${ }^{5}$ Pluvial.
} 
La quantité de pluie a beaucoup diminué pour $41 \%$ des agriculteurs. Pour preuve, ils avancent la réduction des cycles d'irrigation et l'augmentation des besoins en eau des cultures. Auparavant, les agriculteurs irriguaient tous les 15 jours, actuellement, il faut irriguer tous les 4 ou 5 jours.

L'autre manifestation majeure du changement climatique avancée par 35\% des agriculteurs est l'arrivée précoce de l'été, avec des augmentations de température dès les mois d'Avril et de Mai. Ces deux mois sont normalement des mois d'un printemps assez doux. Ces derniers temps, les agriculteurs ont observé qu'ils sont plutôt marqués par des chaleurs inhabituelles.

La perturbation des saisons est un autre signe du changement climatique que $11 \%$ des enquêtés ont observé. Pour eux, il n'y a plus que deux saisons: l'été et l'hiver. Ces agriculteurs versent dans le même sens que les premiers. L'irrégularité de la pluviométrie est relevée par $6 \%$ des agriculteurs, qui estiment qu'il y a «soit beaucoup de pluie, soit qu'il n'y en a pas du tout».

Le sentiment dominant est que les changements sont tels que les agriculteurs vivent dans la plus grande des incertitudes et qu'ils ne peuvent rien prévoir en matière de pluviométrie. Les précipitations étaient, par le passé, et de par leur vécu personnel, plus importantes et plus assurées. Sans trop de soucis, les agriculteurs labouraient dès l'approche de l'automne. Actuellement ils attendent les premières pluies pour le faire, craignant l'absence de la pluie ou son arrivée tardive. C'est un changement de comportement indéniable que certains agriculteurs attribuent à une faiblesse de cette «foi indéfectible» appelée, Niyya. Les agriculteurs se remettent moins facilement à Dieu et sont plutôt attentifs aux prévisions de la météo.*

\subsection{Perceptions changement climatique sur l'activité agricole des effets du}

Les agriculteurs enquêtés sont conscients des effets du changement climatique sur leurs activités agricoles. Ils situent ces effets à un double niveaux de perception intimement liés: i) les effets sur les conditions météorologiques de la production agricole, et, ii) les effets sur le système de production agricole.

\subsubsection{Perceptions des effets du changement climatique sur l'activité agricole}

* Effet du changement climatique sur les saisons agricoles

Le changement climatique a provoqué une grande perturbation des saisons agricoles. Pour les enquêtés, comme avancé précédemment, ils n'existent plus que deux saisons: l'été et l'hiver. Cette perturbation va affecter le temps des principaux travaux agricoles, notamment les labours et la récolte.

Les agriculteurs rappelaient qu'autrefois la pluie tombait à des moments opportuns coïncidant avec les saisons des labours et des semailles. L'agriculteur vivait dans une certaine harmonie avec les saisons. Il labourait et semait en automne qui coïncidait avec la saison des pluies. Il récoltait l'été, en début du mois de juillet et l'arrivée des premières chaleurs. La presque totalité des agriculteurs (98\%) sont catégoriques que la saison des moissons des céréales survient de manière plus précoce qu'auparavant.

La perturbation des saisons se traduit par un changement du calendrier des labours et des semailles, par une incertitude quant à l'avènement des pluies et une arrivée précoce de la saison des moissons poussée par l'augmentation des températures qui accélère la maturité des céréales. 
* Effet du changement climatique sur le calendrier agricole

Les activités agricoles sont inscrites dans des saisons et réglées selon un calendrier agraire antique qui définit le temps prescrit pour les effectuer. Ce calendrier divise les quatre saisons en périodes de temps dites Manazil, au pluriel, et Manzila, au singulier. Ce sont les mansions agricoles. Les mansions les plus connues sont Lyali et Smayam, d'une durée de 40 jours chacune et caractérisées, la première par son froid glacial et son temps pluvieux et la seconde par ses chaleurs caniculaires.

Les agriculteurs qui se sont exprimés lors des «entretiens de groupe»ont été affirmatifs quant à l'effet perturbateur du changement climatique sur le calendrier agricole. Tout à fait le contraire des résultats des enquêtes auprès des agriculteurs, pris isolément, qui montre que seulement $28 \%$ des agriculteurs (tab.2) établissement ce lien. Plus explicitement, ils estiment que la mansion, Manzila, dite Lyali a connu, certes, une diminution des quantités de pluie. Mais 64\% d'entre eux n'ont pas reconnu l'effet du changement sur le calendrier agricole. Quant aux jeunes agriculteurs âgés de moins de 35 ans, et représentant $8 \%$ des enquêtés, ils estiment que ce calendrier n'a plus cours pour orienter leurs activités agricoles.

Cette forme de connaissance relative aux relations mansions agricoles/ saisons agricoles, identifiés chez les personnes âgées, est en perte de vitesse. C'est pourquoi ce résultat demande à être relativiser sachant que dans le précédent paragraphe presque tous les agriculteurs enquêtés en groupe ont reconnu l'effet du changement climatique sur les saisons des travaux agricoles.

Tableau 2. Effet du changement de la pluviométrie sur le calendrier agricole

\begin{tabular}{|l|c|c|}
\hline \multicolumn{1}{|c|}{ Effets } & Nombre de réponses & Pourcentage \\
\hline Pas d'effet & 32 & $64 \%$ \\
\hline Diminution de pluie pendant ''Lyali' & 14 & $28 \%$ \\
\hline Ce calendrier n'a plus cours & 4 & $8 \%$ \\
\hline Total & 50 & $100 \%$ \\
\hline
\end{tabular}

Source: Enquêtes, 2017

\subsubsection{Effets sur le système de production agrícola}

Le changement climatique a produit, selon les agriculteurs, de nombreux effets sur le système de production de la petite région et du Douar enquêté. Le changement climatique est identifié et imputé à trois principaux facteurs de changement, la pluviométrie, la température et l'enneigement. Les agriculteurs apprécient différemment les effets de ces trois facteurs sur leurs systèmes de culture et d'élevage.

* Effet du changement climatique sur le système de culture

Les principaux facteurs du changement climatique identifiés par les agriculteurs ont provoqué huit effets sur les cultures pratiquées (Tab.3). Mais c'est la pluviométrie qui est retenue comme la cause majeure produisant le plus d'effets sur le système de culture. 
26| Perceptions du changement climatique et pratiques adaptatives des agriculteurs du Sais (Maroc)

Tableau 3. Effets du changement climatique sur le système de culture

\begin{tabular}{|l|c|c|c|}
\hline \multirow{2}{*}{ Types d'effets } & \multicolumn{3}{|c|}{ Facteurs de changement } \\
\cline { 2 - 4 } & $\begin{array}{c}\text { Nombre réponse } \\
\text { Pluviométrie }\end{array}$ & $\begin{array}{c}\text { Nombre } \\
\text { réponse } \\
\text { Température }\end{array}$ & $\begin{array}{c}\text { Nombre } \\
\text { réponse } \\
\text { Enneigement }\end{array}$ \\
\hline Diminution de la production & 42 & 0 & 0 \\
\hline Diminution du rendement & 28 & 0 & 0 \\
\hline Apparition de maladies & 10 & 15 & 0 \\
\hline $\begin{array}{l}\text { Dégradation de la qualité des } \\
\text { produits }\end{array}$ & 6 & 0 & 0 \\
\hline $\begin{array}{l}\text { Changement dans la rotation } \\
\text { des cultures }\end{array}$ & 2 & 0 & 0 \\
\hline $\begin{array}{l}\text { Augmentation des besoins en } \\
\text { eau }\end{array}$ & 0 & 38 & 0 \\
\hline $\begin{array}{l}\text { Diminution de l'humidité du } \\
\text { sol }\end{array}$ & 0 & 0 & 32 \\
\hline $\begin{array}{l}\text { Neige sèche brule les produits } \\
\text { agricoles }\end{array}$ & 0 & 0 & 9 \\
\hline \multicolumn{1}{|c|}{ Total } & 88 & 53 & 41 \\
\hline
\end{tabular}

Source: Enquêtes, 2017

Les 50 agriculteurs interrogés estiment, avec 88 occurrences, que c'est le changement de la pluviométrie, qui a causé le plus d'effet sur les cultures, suivi du changement de la température évoqué 53 fois par les agriculteurs et enfin la baisse de l'enneigement citée 41 fois. Le nombre de réponse dépasse le nombre d'agriculteurs enquêtés car un même agriculteur peut fournir plus d'une réponse.

Quant aux effets de ces facteurs du changement climatique sur les cultures pratiquées, ce sont respectivement la baisse de la production (42 occurrences), l'augmentation des besoins en eau des cultures (citée 38 fois), la diminution de l'humidité des sols (citée 32 fois) et la baisse du rendement (citée 28 fois) qui sont revenus le plus souvent dans leurs réponses. D'autres effets sont cités, comme l'apparition des maladies causée par le changement de la pluviométrie (10 citations) et de la température (15 citations).

La baisse de la pluviométrie et son irrégularité sont données comme cause de la diminution de la production par 42 agriculteurs, suivie de la diminution des rendements, citée par 28 agriculteurs, puis de l'apparition des maladies évoquée par 10 agriculteurs. Ces derniers déclarent qu'avec l'absence des pluies, leurs cultures sont plus exposées au développement de nouvelles maladies comme le manque de cuivre, le feu bactérien ... Selon leurs dires, l'eau de la pluie confère une résistance aux cultures.

L'irrégularité de la pluviométrie a affecté également la qualité de quelques produits agricoles, selon 6 agriculteurs. A titre d'exemple, ils disent que la pluie qui tarde à tomber au mois de Mai affecte la qualité du blé. Cette irrégularité a perturbé aussi la rotation de leurs cultures, car ils ont abandonné certaines cultures (comme l'ail) qui rentraient dans les rotations des cultures appliquées.

L'augmentation de la température a, par ailleurs, eu pour effet l'augmentation des besoins en eau des cultures, selon 38 agriculteurs enquêtés, et l'apparition des maladies, selon 15 d'entre eux. 
La baisse de l'enneigement, a provoqué, selon les agriculteurs, deux principaux effets. D'un côté, la baisse de l'humidité du sol qui est due à la diminution de la quantité de neige. La neige conserve l'humidité dans le sol affirment 32 agriculteurs. De l'autre côté, l'apparition de la neige dite «sèche» qui «brûle» les produits agricoles. C'est un effet cité par 9 agriculteurs qui ont constaté qu'il y a un changement dans la qualité de la neige. Celle-ci est devenue sèche, c'est-à-dire qu'elle ne contient pas assez d'eau, et, par conséquence, elle brûle les feuilles des cultures, l'olivier notamment.

*Effet du changement climatique sur le système d'élevage

Les facteurs du changement climatique et leurs effets sur les systèmes d'élevage sont représentés dans le tableau 4. Les agriculteurs ont identifié quatre effets du changement climatique sur le système d'élevage, qui sont respectivement: l'appauvrissement des parcours (91 occurrences), le recours à l'achat des aliments de bétail ( 5 occurrences), la cherté des aliments du bétail ( 2 occurrences), et en dernier lieu un seul agriculteur qui a lié la vulnérabilité de ses animaux à l'augmentation de la température.

Tableau 4. Effets du changement climatique sur le système d'élevage

\begin{tabular}{|l|c|c|c|}
\hline \multirow{2}{*}{ Types d'effets } & \multicolumn{3}{|c|}{ Facteurs du changement } \\
\cline { 2 - 4 } & $\begin{array}{c}\text { Pluviométrie } \\
\text { Nbre réponse }\end{array}$ & $\begin{array}{c}\text { Température } \\
\text { Nbre réponse }\end{array}$ & $\begin{array}{c}\text { Enneigement } \\
\text { Nbre réponse }\end{array}$ \\
\hline Appauvrissement des parcours & 45 & 33 & 13 \\
\hline Recours à l'achat des aliments de bétail & 5 & 0 & 0 \\
\hline Cherté des aliments du bétail & 0 & 0 & 2 \\
\hline Animaux vulnérables aux maladies & 0 & 1 & 0 \\
\hline
\end{tabular}

Source: Enquêtes, 2017.

Par le passé, l'alimentation du cheptel était basée sur les parcours, constamment riches en herbes en raison de l'abondance de la pluie. ${ }^{6}$ Mais actuellement, selon 45 agriculteurs, les parcours ont été dégradés à cause de la diminution des précipitations. Selon 5 autres agriculteurs, cette diminution a poussé les éleveurs à acheter les aliments de bétail qui, par ailleurs, sont devenus de plus en plus chers. 33 agriculteurs pensent que l'augmentation de la température est la cause de l'appauvrissement des parcours et de leur dégradation. Les effets de la baisse des chutes de neige sur le système d'élevage ont été reconnus par 13 enquêtés qui estiment que la diminution de la neige est la cause de l'appauvrissement des parcours. Pour eux, la neige humidifie le sol et l'herbe pousse plus facilement. Ce qui n'est plus le cas maintenant.

\subsection{Pratiques d'adaptation au Changement Climatique}

Les agriculteurs se sont révélés bien conscients du changement climatique. Ils parviennent à identifier les facteurs le provoquant ainsi que leurs effets négatifs sur leur système de production agricole. Et pour régler leur conduite sur ce changement, les agriculteurs ont mis en œuvre diverses pratiques d'adaptation en modifiant les composantes de leur système de production.

\footnotetext{
${ }^{6}$ Les agriculteurs enquêtés font partie de communautés constituées anciennement d'éleveurs transhumants.
} 


\subsubsection{Modifications du système de culture}

Les principales pratiques développées par les agriculteurs enquêtés (et ceux de la petite région) consistent en l'abandon de certaines cultures, l'introduction d'autres, le creusement de puits et la diversification des sources de revenu.

* Introduction ou abandon de certaines cultures

$20 \%$ des agriculteurs ont abandonné certaines cultures et/ou introduit d'autres. Les cultures sacrifiées sont celles qui sont les plus exigeantes en eau. Elles sont remplacées par des cultures plus résistantes au stress hydrique ou par des variétés d'arbres qui n'exigent pas de froid. C'est l'exemple de 3 agriculteurs qui ont introduit une nouvelle variété de pommier «Royal Guala» qui supporte mieux les fortes températures.

* Creusement des puits et installation du système goutte à goutte

C'est le cas de 16 agriculteurs qui ont creusé des puits et 9 d'entre eux qui ont installé un bassin de stockage de l'eau et un système d'irrigation localisé. C'est le moyen de réduire la dépendance vis à vis des irrégularités de la pluviométrie. Pour réaliser ces investissements, ces agriculteurs ont bénéficié d'une subvention accordée par l'Etat dans le cadre du Fonds de Développement Agricole (FDA).

* Diversification de sources de revenus

La mise en œuvre d'activités complémentaires génératrices de revenus para agricoles est un mécanisme mis en œuvre par les agriculteurs pour assurer la survie de leur famille et pour s'adapter aux changements climatiques. C'est le recours à la pluriactivité bien connu chez les exploitations familiales. Pour 6 agriculteurs, le revenu agricole devient incertain à cause de la contrainte climatique. L'exercice d'une autre activité dans le commerce, le transport, le bâtiment, etc., est une alternative pour en atténuer les effets du changement climatique. Sur les 50 agriculteurs enquêtés, 12 reconnaissent recevoir des aides financières de leurs fils qui travaillent en dehors de l'exploitation. Ces aides sont considérés importantes et leur permettent d'atténuer les effets climatiques sur leur revenu agricole.

* Retour à la piété

Deux agriculteurs ont répondu que la meilleure façon de s'adapter au changement climatique est d'effectuer un retour à la piété. Ils pensent que le changement climatique est, tout simplement, une punition de Dieu. Dans leur conception, les gens doivent suivre le chemin de Dieu et éduquer les générations futures à mieux le faire.

\subsubsection{Modifications du système d'élevage}

Les principales pratiques adaptatives en matière d'élevage consistent soit à diminuer la taille des troupeaux soit à abandonner l'activité d'élevage.

* Diminution d'effectif et achat des aliments

D'après les réponses des agriculteurs, les espaces de parcours se sont beaucoup réduits à cause du changement climatique. L'herbe ne pousse plus en montagne comme auparavant. Par le passé, le bétail pouvait se nourrir sur les parcours pendant 8 à 9 mois par an. Actuellement, il faut lui assurer sa nourriture à partir des aliments achetés tout au long de l'année. Ces conditions ont poussés 36 agriculteurs qui pratiquent l'élevage, soit $72 \%$ de l'échantillon, à diminuer l'effectif de leur cheptel pour pouvoir maitriser l'achat des aliments: «Il faut sacrifier 10 brebis pour faire vivre 100 autres», dit un agriculteur. 
* Abandon de l'élevage

A cause de la dégradation du parcours qui constituait la principale source d'alimentation du cheptel, 7 agriculteurs de l'échantillon ont tout simplement abandonné l'élevage. Ils affirment qu'ils n'ont pas les moyens d'acheter les aliments devenus incontournables.

\subsection{Opinions sur les liens entre facteurs du changement et pratiques adaptatives}

Les entretiens de groupe ont révélé une série de pratiques adaptatives aux trois facteurs du changement climatique identifiés par les agriculteurs, à savoir, la pluviométrie, la température et l'enneigement. C'est une panoplie de pratiques adaptatives (Tb.5) qui a été proposée aux agriculteurs enquêtés pour recueillir leurs opinions sur le lien existant entre ces pratiques et les facteurs de changement.

\subsubsection{Pratiques adaptatives et changement de la pluviométrie}

L'ensemble des agriculteurs est tout à fait d'accord que c'est la diminution de la pluviométrie qui a poussé les agriculteurs à creuser des puits et $74 \%$ d'entre eux (Tab.5) estiment que cette même raison qui a incité les agriculteurs de la région à modifier les règles de gestion coutumières des eaux d'irrigation pour les rendre plus efficientes.

La diminution de la pluviométrie est, pour 92\% des agriculteurs, la cause de l'introduction des nouvelles variétés de céréales résistantes au stress hydrique. Pour $42 \%$ des enquêtés, cette même diminution est à l'origine de l'introduction de nouvelles espèces fruitières qui s'adaptent mieux à la chaleur et à l'irrégularité de la pluviométrie. En revanche, pour une partie des agriculteurs des plus âgés, soit $18 \%$, des variétés qui s'adaptent à la diminution de pluviométrie n'existent pas, car toutes les cultures ont besoin d'eau. Le changement d'assolement et de rotation des cultures est une pratique adaptative qui est impulsée par la diminution de la pluviométrie reconnaissent $60 \%$ des agriculteurs.

Tableau 5. Pratiques adaptatives et changement de la pluviométrie

\begin{tabular}{|c|c|c|c|c|c|c|c|c|}
\hline \multirow{3}{*}{$\begin{array}{c}\text { Pratiques } \\
\text { adaptatives }\end{array}$} & \multicolumn{8}{|c|}{ Opinions des agriculteurs } \\
\hline & \multicolumn{2}{|c|}{$\begin{array}{c}\text { Tout à fait } \\
\text { d'accord }\end{array}$} & \multicolumn{2}{|c|}{ D'accord } & \multicolumn{2}{|c|}{ Pas d'accord } & \multicolumn{2}{|c|}{ Sans Opinion } \\
\hline & Nombre & $\%$ & Nombre & $\%$ & Nombre & $\%$ & Nombre & $\%$ \\
\hline $\begin{array}{l}\text { Irrigation } \\
\text { par eaux } \\
\text { souterraines }\end{array}$ & 50 & $100 \%$ & 0 & 0 & 0 & 0 & 0 & 0 \\
\hline $\begin{array}{l}\text { Introduction } \\
\text { nouvelles variétés } \\
\text { céréales }\end{array}$ & 46 & $92 \%$ & 0 & 0 & 3 & $6 \%$ & 1 & $2 \%$ \\
\hline $\begin{array}{l}\text { Changement règles } \\
\text { gestion sociale eaux } \\
\text { d'irrigation }\end{array}$ & 37 & $74 \%$ & 8 & $16 \%$ & 6 & $\begin{array}{l}12 \\
\% \\
\end{array}$ & 5 & $10 \%$ \\
\hline $\begin{array}{l}\text { Abandon parcours } \\
\text { et achat aliments } \\
\text { bétail }\end{array}$ & 49 & $98 \%$ & 1 & $2 \%$ & 0 & 0 & 0 & 0 \\
\hline $\begin{array}{l}\text { Changement } \\
\text { rotation cultures }\end{array}$ & 32 & $64 \%$ & 18 & $36 \%$ & 0 & & 0 & 0 \\
\hline $\begin{array}{l}\text { Changement } \\
\text { assolement }\end{array}$ & 33 & $66 \%$ & 15 & $30 \%$ & 2 & $4 \%$ & 0 & 0 \\
\hline $\begin{array}{l}\text { Introduction } \\
\text { nouvelles espèces } \\
\text { fruitières }\end{array}$ & 21 & $42 \%$ & 3 & $6 \%$ & 9 & $\begin{array}{l}18 \\
\%\end{array}$ & 17 & $34 \%$ \\
\hline
\end{tabular}

Source: Enquêtes, 2017. 


\subsubsection{Pratiques adaptatives et changement de la température}

Le changement de température serait-il à l'origine des pratiques adaptatives identifiées (Tb.6) La presque totalité des agriculteurs (90\%) approuvent que c'est bien l'augmentation de température qui a été à l'origine du délaissement du pommier, qui exige un nombre important de jours de froid par année, et de l'introduction de nouvelles variétés de céréales qui supportent un haut degré de température.

La moitié des agriculteurs sont d'accord qu'il y a de nouvelles espèces fruitières introduites qui s'adaptent mieux au changement climatique. Et c'est ce qui explique l'orientation de certains agriculteurs de la petite région à s'orienter vers l'arboriculture. Cependant, $30 \%$ des agriculteurs enquêtés n'ont aucune idée sur l'existence de ces espèces résistantes.

L'augmentation de la température a été, pour presque la totalité des agriculteurs, à l'origine de l'abandon de certaines cultures exigeantes en eau, comme l'ail, l'oignon, pour certains, et par conséquence, de changer la rotation de leurs cultures.

Tableau 6. Pratiques adaptatives et changement de la température

\begin{tabular}{|l|c|c|c|c|c|c|}
\hline \multirow{2}{*}{ Pratiques adaptatives } & \multicolumn{6}{|c|}{ Opinions des agriculteurs } \\
\cline { 2 - 7 } & \multicolumn{3}{|c|}{ Oui } & \multicolumn{2}{c|}{ Non } & \multicolumn{2}{c|}{ Ne sais pas } \\
\cline { 2 - 7 } & Nombre & $\%$ & Nombre & $\%$ & Nombre & $\%$ \\
\hline Délaissement du pommier & 48 & $96 \%$ & 0 & $0 \%$ & 2 & $4 \%$ \\
\hline $\begin{array}{l}\text { Introduction nouvelles variétés } \\
\text { céréales }\end{array}$ & 46 & $92 \%$ & 3 & $6 \%$ & 1 & $2 \%$ \\
\hline $\begin{array}{l}\text { Introduction nouvelles espèces } \\
\text { fruitières }\end{array}$ & 29 & $58 \%$ & 6 & $12 \%$ & 15 & $30 \%$ \\
\hline Changement rotation cultures & 49 & $98 \%$ & 1 & $2 \%$ & 0 & $0 \%$ \\
\hline Changement assolement & 49 & $98 \%$ & 1 & $2 \%$ & 0 & $0 \%$ \\
\hline
\end{tabular}

Source: Enquêtes, 2017.

\subsubsection{Pratiques adaptatives et changement de l'enneigement}

Les agriculteurs interrogés sont unanimes quant à la régression des chutes des neiges dans la région. Cette régression serait, pour 52\% des enquêtés (tab.7), la raison de l'introduction des nouvelles cultures, et pour 38\% d'entre eux, du changement de rotation et d'assolement des cultures.

Tableau 7. Pratiques adaptatives et changement de l'enneigement

\begin{tabular}{|l|c|c|c|c|}
\hline \multirow{2}{*}{ Comportements adaptatifs } & \multicolumn{3}{c|}{ Opinions des agriculteurs } \\
\cline { 2 - 5 } & \multicolumn{3}{|c|}{ Oui } & \multicolumn{2}{c|}{ Non } \\
\cline { 2 - 5 } & Nombre & $\%$ & Nombre & $\%$ \\
\hline Introduction des nouvelles cultures & 26 & $52 \%$ & 24 & $48 \%$ \\
\hline Changement rotation cultures & 1 & $2 \%$ & 49 & $98 \%$ \\
\hline Changement assolement & 1 & $2 \%$ & 49 & $98 \%$ \\
\hline Totaux & 28 & & 122 & \\
\hline
\end{tabular}

Source: Enquête, 2017. 


\section{Conclusion}

Cet article sur les perceptions des changements climatiques, de leurs effets sur les activités agricoles et les pratiques adaptatives développées par les agriculteurs a permis d'identifier les principaux facteurs du changement climatique et leurs manifestations. L'univers de perception des changements des agriculteurs enquêtés est structuré autour de trois facteurs : i) la pluviométrie, qui se manifeste par la diminution et l'irrégularité de la pluie et son arrivée tardive, ii) la température, qui se fait connaitre par sa hausse et par l'arrivée précoce de l'été, iii) l'enneigement qui se raréfie. Le changement climatique a produit ses effets sur: i) Les conditions météorologiques d'exercice de l'activité agricole qui ont perturbé les saisons agricoles et rendu quasi obsolète l'usage du calendrier agraire antique, ii) les systèmes de production agricole, que les agriculteurs ont dû modifier dans leur composantes de culture et d'élevage pour s'adapter à ces changements.

Les pratiques adaptatives développées consistent en: i) l'introduction ou l'abandon de certaines cultures, ii) la limitation de la dépendance de la pluviométrie par le creusement des puits et l'introduction d'équipement hydraulique, iii) la diminution des effectifs du cheptel ou son abandon.

Ces mesures d'adaptations ont consisté en une combinaison d'actions spécifiques (par ex. le changement d'espèce cultivée) et de changements plus ou moins systémiques (par ex. la diversification des moyens d'existence face aux risques). En outre, certaines d'entre elles ont conduit au renforcement des capacités de réaction des agriculteurs comme la mise en place de l'irrigation goutte-à-goutte pour une utilisation efficiente de l'eau et la modification des pratiques agricoles (éviter les monocultures, modifier le calendrier des activités agricoles et avancer les dates de semis pour compenser les contraintes d'humidité pendant les périodes chaudes). Elles sont aussi diverses pour permettre aux agriculteurs d'être dans des situations de «multirationnalité» qui permettraient une adaptabilité plus grande (Sfez et Cauquelin, 2005).

Par ailleurs, ces différentes pratiques adaptatives témoignent des potentialités des «forces cognitives locales» (Pinton et Grenand, 2007) à enrayer une situation de vulnérabilité ou de risque environnemental. Pour faire face aux effets du CC, les agriculteurs enquêtés ont mobilisé différentes compétences résultant d'un savoir-faire local acquis de leur expérience et hérité de leurs aïeuls. Ce qui prouve que la validité des options d'adaptation face à certaines conséquences du changement climatique n'est pas appréciable isolément des usages et valeurs locales (Bertrand et Richard, 2012). En fait, nombreux travaux menés sur l'adaptation mettant en évidence le rôle central des facteurs socioculturels dans les processus locaux d'adaptation (Sfez et Cauquelin, 2005; Magnan, 2009; Breda, 2019).

Toutefois, ces pratiques relèvent encore d'une adaptation spontanée visant à réduire la vulnérabilité des agriculteurs et de leurs exploitations agricoles aux effets actuels du changement climatique. Aussi cherchent-ils à sécuriser et leur alimentation et leur revenu. Ils auront, avec l'accompagnement d'autres acteurs (services d'encadrement, de recherche et de la société civile), à s'inscrire dans une perspective proactive et mettre en place des actions d'adaptation anticipative leur permettant d'accroitre leurs capacités 
32| Perceptions du changement climatique et pratiques adaptatives des agriculteurs du Sais (Maroc)

d'adaptation $^{7}$ aux effets actuels et futurs du changement climatique. Cet accompagnement est à renforcer par une éducation à l'environnement intégrant la dimension genre (Esteban, Amador, Mateos y Olmedo, 2019; Iñigo, 2019) au niveau des territoires ruraux.

\section{Références bibliographiques}

Adger, W.N., Armell, N.W., et Tomppkins, E.L. (2005). Successful adaptation to climate change across scales, Global Environmental Change, (15), 77-86.

Aziz, L., et Elquaoumi, I. (2016). Analyse des stratégies d'adaptations des agriculteurs de Skoura (Ouarzazate) aux changements climatiques. Alternatives Rurales, (4), 38-48.

Aziz L., et Sadok, W. (2015). Stratégies d'adaptation des producteurs du Safran de Taliouine (Maroc) face au changement climatique, Journal of Alpine Research $\mid$ Revue de géographie alpine. [En ligne], 103-2 |2015, mis en ligne le 04 septembre 2015, consulté le 06 Juin 2020. DOI : https://doi.org/10.4000/rga.2820

Bertrand, F., et Richard, E. (2012). Les initiatives d'adaptation aux changements climatiques, Entre maintien des logiques de développement et renforcement des coopérations entre territoires. Territoire en mouvement Revue de géographie et aménagement [En ligne], 14-15 | 2012, mis en ligne le 01 juillet 2014, consulté le 22 juin 2020. DOI: https://doi.org/10.4000/tem.1799.

Breda, N. (2019). Gender, Children and Anthropology in the face of Climate Change. Collectivus, Revista de Ciencias Sociales, 6(1), 197-214. DOI: http://dx.doi.org/ 10.15648/Coll.1.2019.11

CIRAD. (2009). Changement climatique et agriculture : L'environnement et la sécurité alimentaire en jeu. Montpellier, 39.

Dugué, M.J. (2012). Caractérisation des Pratiques d'Adaptation au Changement Climatique en Agriculture Paysanne. Agronome et Vétérinaires sans frontières.

Esteban, M., Amador, L.V., Mateos, F., et Olmedo, F.J. (2019). Les femmes et l'environnement. Une approche de l'action socio-éducative. Collectivus, Revista de Ciencias Sociales, 6(1), 177-195. https://doi.org/10.15648/Coll.1.2019.10

FAO. (2013). Guidelines to control water pollution from agriculture in China: decoupling water pollution from agricultural production. Rome.

GIEC. (2014). 5eme rapport de GIEC sur « le changement climatique et leurs évaluation» (partie 2 : impact, adaptation et vulnérabilité).

GIEC. (2013). Changements climatiques 2013. Les éléments scientifiques. Contribution du Groupe de travail I au cinquième Rapport d'évaluationdu Groupe d'Expert Intergouvernemental sur l'Evolution du Climat. https://www.ipcc.ch/site/assets/uploads/2018/03/ WG1AR5_SPM_brochure_fr.pdf.Consultéle 15/02/2017.

\footnotetext{
${ }^{7}$ La capacité d'adaptation est la triple faculté de réduire la sensibilité du système, d'en modifier l'exposition et d'en accroître la résilience (Adger et al., 2005).
} 
Iñigo, L. (2019). Éducation pour la préservation de l'environnement. Collectivus, Revista de Ciencias Sociales, 6(1), 215-234. DOI: https://doi.org/10.15648/Coll.1.2019.12

Kamil, H. (1999). Elevage nomade, appropriation de l'espace et structuration chez les Ouled Khawa. These de doctorat: Lettres : Aix-Marseille 1 - France.

Magnan, A. (2009). Proposition d'une trame de recherche pour appréhender la capacité d'adaptation aux changements climatiques. VertigO - la revue électronique en sciences de l'environnement, Volume 9 (Numéro 3), décembre 2009, [en Ligne : http://vertigo .revues.rog/9189].

Ministère de l'Intérieur. (2015). Monographie générale de la Région Fès-Meknès.57.

Pinton, F., et Grenand, P. (2007). Savoirs traditionnels, populations locales et ressources globalisées. In : Aubertin C., Pinton F. et Boisvert V., Les marchés de la biodiversité, IRD Éditions, Paris.

Sfez, L., et Cauquelin, A. (2005). Analyse des attitudes face à l'adaptation au changement climatique : le cas de deux stations de moyenne montagne dans les Alpes-de-HauteProvence », pp.99-108 in TERRITOIRES 2030, Changement climatique, énergie et développement durable des territoires, décembre 2005 n² $^{\circ}$, DATAR, Paris, 114.

Vissoh, P.V. et al. (2012). Perceptions et pratiques d'adaptation aux changements climatiques : le cas des communes d'Adjohoun et de Dangbo au Sud-Est Bénin. Les Cahiers d'Outre-Mer, (260), 479-492.

Rishi, P., et Mudaliar, R. (2014). L'échelle de perception du changement climatique, cité in: Forum de la Méditerranée, 18 Mars 2016. La Perception du Changement Climatique et le bien-être sur la Méditerranée française : enseignements de Marseille et Nice. 